\title{
El riesgo perinatal y materno del embarazo gemelar
}

JORGE HASBÚN(1)

\section{RESUMEN}

En relación con el aumento de la gemelaridad en los últimos 20 años, se revisan las complicaciones del embarazo gemelar, las limitaciones de la data epidemiológica, los riesgos perinatales de mortalidad, prematurez, daño neurológico y el riesgo materno por complicaciones graves. Se discute el embarazo de alto riesgo y el uso de Técnicas de Reproducción Asistida.

Palabras clave: riesgo perinatal, riesgo materno, embarazo gemelar.

\section{ABSTRACT \\ PERINATAL AND MATERNAL RISK IN TWIN PREGNANCY}

In relation to the increase of twin births during the last 20 years, complications of twin births are being checked, as well as the limitations of epidemiological data, perinatal mortality risks, prematurity, neurological damage, and maternal risk due to serious complications. High rank pregnancies and the use of Assisted Reproduction Techniques are discussed.

Key words: perinatal risk, maternal risk, twin pregnancy.

\section{INTRODUCCIÓN}

El propósito del documento es revisar la importancia epidemiológica y clínica del embarazo múltiple en relación con la incorporación de nuevas técnicas en el manejo de la infertilidad.

La disminución de la gemelaridad espontánea en el curso del siglo XX por el uso de anticonceptivos, se revirtió, duplicándose su incidencia en los últimos 20 años y triples o mayores aumentaron siete veces en relación con únicos ${ }^{1}$. La primera causa es el tratamiento moderno de la infertilidad mediante Inducción de Ovulación (clomifeno, gonadotropinas), o con el uso de Técnicas de Reproducción Asistida (TRA): transferencia de embriones obtenidos por fertilización in vitro, transferencia intratubaria de gametos o zigotos y procedimientos complementarios.

Estas técnicas son muy efectivas y están ampliamente disponibles, pero son de alto costo y su uso ha generado una gemelaridad iatrogénica de mayor morbimortalidad perinatal y que aumentó la relación gemelos espontáneos/inducidos de 1: 46 en la década 70, a $1: 2$ en $\operatorname{los} 90^{2}$. Es difícil de evaluar porque no hay certificación específica de nacimientos gemelares ni de los obtenidos por Inducción de Ovulación. Con el uso de TRA, en centros

(1) Departamento de Obstetricia y Ginecología. Unidad Medicina Materno-Fetal. Hospital Clínico U. de Chile. jorgehasbun@latinmail.com 
desarrollados se estima que genera $44 \%$ de gemelos y $12 \%$ de triples ${ }^{3}$.

El segundo factor es el aumento actual de embarazos en edades más tardías lo que se asocia a mayor gemelaridad espontánea y a fecundidad reducida 4 , generando tratamientos con TRA. Además las gestantes al final de la vida reproductiva son más comúnmente portadoras de enfermedades subyacentes y hacen complicaciones maternas gestacionales más graves ${ }^{5}$.

\section{LIMITACIONES DE LA DATA GEMELAR}

La incidencia tiene un subregistro porque no hay información hospitalaria del aborto gemelar o pérdida fetal temprana. El pronóstico está influido por el bajo poder estadístico de series con pocos casos; por inclusión o no de mal formados; por el ajuste de peso; por cambios en la conducta médica; por descenso reciente de la mortalidad que beneficia tanto a gemelos como a únicos, y por factores propios del parto (vía, orden del nacimiento, manejo de distocias), variables confundentes difíciles de controlar y con resultados conflictivos de manejo.

El incremento de embarazos gemelares justifica una visión integral del riesgo en la patología obstétrica más compleja, y una mejor comprensión en los alcances del cuidado médico. Hay un espectro de complicaciones graves de expresión variable, aditivas en riesgo continuo y una limitación frecuente es encasillarlas como fetales o maternas, subestimando su período de ocurrencia e interacción.

En el período antenatal aumenta la posibilidad de metrorragia, anemia e hipertensión materna, polihidroamnios, parto prematuro, muerte fetal y de un subgrupo de riesgos propios de gemelos monocoriónicos en II y III trimestre por comunicaciones vasculares placentarias.

Los riesgos intraparto incluyen parto distócico, complicaciones del cordón umbilical, hipoxia aguda, gemelos en colisión y cesárea de urgencia. En el post-parto hay mayor riesgo de hemorragia por inercia uterina, depresión materna y complicaciones de cesárea.

En el período neonatal hay riesgos por prematurez, asfixia intraparto, bajo peso de nacimiento, restricción fetal, manejo de malformaciones y complicaciones de gemelos monocoriónicos.

El cuidado médico es de gran interés, pero un primer objetivo es comparar el pronóstico de gemelos con únicos e interpretar el impacto epidemiológico de la mayor incidencia actual.

1. Mortalidad perinatal (MPN): 1 - $2 \%$ en únicos, aumenta a $8-10 \%$ en gemelos. Si bien gemelos representan $2,6 \%$ del total de partos, su contribución relativa a la MPN global es de $12-14 \%{ }^{6}$ ( Tabla 1 ).

Tabla 1. Pronóstico

\begin{tabular}{|lcr|}
\hline & $\begin{array}{c}\text { MORTALIDAD } \\
\text { PERINATAL }\end{array}$ & $\begin{array}{r}\text { MORTALIDAD } \\
\text { NEONATA }\end{array}$ \\
UNICOS & $1-2 \%$ & $1-2 \%$ \\
GEMELOS & $8-10 \% *$ & $7 \% * *$ \\
$*$ & MPN Global : 12-14\% por gemelar \\
$* *$ & RR 6,6 (ref 7 )
\end{tabular}

Al controlar las variables edad gestacional y peso, las tasas de mortalidad específicas por categoría de peso son similares para gemelos y únicos, con menos de $2500 \mathrm{~g}$; pero a pesos superiores la mortalidad es mayor en gemelos ${ }^{7-8}$.

La mortalidad neonatal es de $1,2 \%$ en únicos $\mathrm{y}$ asciende a $7 \%$ en gemelos con un mayor riesgo relativo de 6,6. También esta tasa es más alta en gemelos que en únicos a pesos mayores de $2500 \mathrm{~g}^{7-8}$.

Aún cuando la prematurez es la causa principal de MPN en gemelos (80\%), hay una mayor mortalidad en gemelos $>$ de $2500 \mathrm{~g}$ con respecto a únicos de igual peso. La mayor maduración placentaria de gemelos ${ }^{9}$, integrada a las causas de muerte en gemelos maduros y grandes ${ }^{10-11}$ sugiere que el término de la gestación ocurre en semana 38 y a diferencia del único, semanas 39 a 41, definirían el gemelar post-término o prolongado lo cual tiene importancia clínica. 
2. Prematurez gemelar: $44,2 \%$ hacen parto prematuro y su importancia es que genera globalmente el $12 \%$ de muertes perinatales, $7 \%$ de las muertes neonatales y alta morbilidad en sobrevivientes 12-14.

Las características de la Prematurez gemelar en el Hospital Clínico U de Chile seleccionando todos los prematuros, gemelos y únicos, entre semanas 26-36 demostró que en gemelares fue de $50 \%$ versus $13 \%$ en únicos y que en su contribución relativa al total de prematuros, los gemelos aportan $20.8 \%$ en semanas $24-28$, $12.8 \%$ en semanas $29-33$ y $10.8 \%$ en semanas 34 -36 (Tabla 2). (Libro de Resúmenes Congreso Chileno de Obstetricia y Ginecología 2001, p 84-87).

Tabla 2. Contribución relativa de la gemelaridad a la prematurez

\begin{tabular}{|lccrc|}
\hline & $\begin{array}{c}\text { PREMATUROS } \\
\text { UNICOS }\end{array}$ & \multicolumn{2}{c|}{ PREMATUROS } \\
& GEMELOS \\
Semana $24-28$ & 48 & 10 & $(20.8 \%)$ \\
Semana $29-33$ & 163 & 21 & $(12,9 \%)$ \\
Semana $34-36$ & 1266 & 135 & $(10.8 \%)$ \\
\hline
\end{tabular}

Se calculó la frecuencia de prematuros para cada semana gestacional y se construyó una curva comparativa para gemelos y únicos con la edad al parto ( Figura 1) .

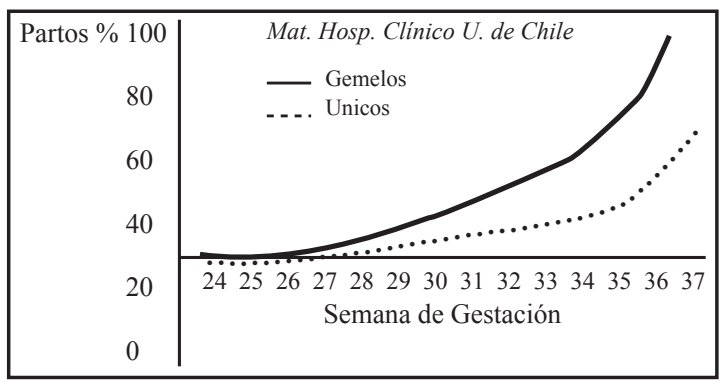

Figura 1. Partos prematuros gemelares v/s partos prematuros únicos.

La edad gestacional comparada al parto de prematuros únicos y gemelos, según nacimientos acumulados cada semana demostró que la prematurez gemelar es más frecuente y precoz que en únicos.

La etiología más frecuente de la prematurez gemelar es el comienzo espontáneo del parto sin un factor desencadenante y luego preeclampsia e hipertensión materna, restricción fetal, desprendimiento placentario y complicaciones de placenta monocoriónica. Factores como sobredistensión uterina15, coriamnionitis subclínica, rotura de membranas $\mathrm{y}$ vaginosis bacteriana16, no han demostrado asociación, pese a ser factores reconocidos de prematurez en únicos.

El embarazo gemelar siguiente a un prematuro único, se asocia un mayor riesgo de prematurez grave: riesgo 2.3 para gemelos en semanas $30-34$ y riesgo 3.06 para gemelos menores de 30 semanas 17 .

Hay consenso epidemiológico que una disminución del parto prematuro puede reducir la morbilidad y mortalidad de los gemelos, pero en el contexto actual de uso de TRA, su contribución irá en aumento.

3. Daño neurológico en gemelos: la Parálisis Cerebral (PC) o discapacidad causada por daño cerebral intrauterino o neonatal está significativamente aumentada en gemelos.

La prevalencia de PC en gemelos es $7.4 \%$, siete veces más que en únicos y se relaciona tanto con el exceso de prematurez como con la gemelaridad misma ${ }^{18}$.

La prevalencia es progresiva con mayor número de fetos: únicos 1.6, gemelos $7.4 \mathrm{y}$ triples 28 por 1000 sobrevivientes al año de vida respectivamente ${ }^{18-20}$.

Series estratificadas que comparan únicos y múltiples demuestran que a pesos menores de $2500 \mathrm{~g}$ la incidencia de PC es similar, en cambio a pesos mayores de $2500 \mathrm{~g}$ hay un aumento significativo en gemelos (3.6 veces) ${ }^{21}$.

La prematurez es el factor de riesgo más importante para $\mathrm{PC}$, similar en únicos y gemelos, sin embargo el riesgo relativo de PC es significativamente mayor en gemelos con más de 37 semanas $^{20-21}$.

En únicos, el riesgo de PC disminuye con el incremento de peso y con la edad gestacional avanzada, en el gemelar paradojalmente el riesgo es mayor y también sugiere que el término de 
la gestación es más temprano que en el embarazo único.

Otros factores que generan daño neurológico son la muerte in útero de un co-gemelo, el tratamiento in útero de la transfusión intergemelar y la gemelaridad iatrogénica multifetal.

4. Cigocidad y placentación: La génesis del riesgo perinatal descrito yace en la cigocidad y la placentación. La gemelaridad dicigota (60$70 \%$ ) ocurre con más de una ovulación y cada óvulo es fertilizado por un espermatozoide. Ocurre en 1\% de los nacimientos, por mayores niveles de hormona foliculoestimulante, en mujeres más altas, de mayor edad y paridad, con historia familiar y herencia racial. En la gemelaridad monocigota $(30 \%)$ un óvulo fecundado, el cigoto, se divide generando monocigóticos. Es una anomalía constante, $3.5 / 1000$ nacimientos, que se produce por trauma bioquímico o mecánico del blastoquiste.

La cigocidad influye en el futuro de los gemelos ya que es importante en el diagnóstico de enfermedad genética o con herencia multifactorial como cardiovasculares, algunos tipos de cáncer, en compatibilidad de transplantes y en cirugía reparadora ${ }^{22-23}$. Se han comunicado en el monocigoto con repetición ulterior en su co-gemelo, lo que abre posibilidades de predicción y anticipación (aún con cirugía sobre áreas precancerosas).

La placentación o corionicidad se asocia estrechamente a la morbimortalidad. Los dicigotos desarrollan una placenta cada uno (bicoriónicos), tienen septum que los separa y funcionan como unidades independientes. Los monocigotos en cambio, pueden ser mono o bicoriónicos (una o dos placentas). Todos los monocigotos con placenta monocoriónica tienen conexiones anastomóticas vasculares intraplacentarias de diferente tipo ${ }^{24}$ que permiten una transfusión bidireccional balanceada ante diferencias de presión arterial intergemelar. La ausencia de una anastomosis compensadora o protectora determina una transfusión unidireccional y crónica, de mal pronóstico perinatal25-26 y explica las complicaciones clínicas mayores: la transfusión gemelo-gemelo, la Transfusión aguda periparto, la Muerte de un gemelo y el Acardio. Este tipo de placentación representa la segunda causa de muerte después de la prematurez.

El monocoriónico tiene mayor mortalidad perinatal $(3 / 1)$, prematurez $(2 / 1)$, restricción del crecimiento fetal (3/1), daño neurológico (3/1) e hipoxia fetal crónica $(5 / 1)$ que bicoriónicos 27-28, asociados al rol de las anastomosis, muy frecuentes en los primeros y excepcionales en bicoriónicos.

Sin embargo los bicoriónicos representan un alto riesgo que también requiere vigilancia especial, ya que es frecuente la restricción selectiva del crecimiento de uno, puede ocurrir muerte de uno in útero y están descritas placentas dicoriónicas fusionadas con anastomosis ${ }^{29}$.

Actualmente la determinación precoz de corionicidad en el primer trimestre, según las características de las membranas con ultrasonido transvaginal (US), es clave en un manejo óptimo, porque estratifica subgrupos de riesgo para vigilancia antenatal específica.

En monocoriónicos el tratamiento es quirúrgico in-útero, con la segregación circulatoria mediante coagulación laser selectiva de las anastomosis en la placenta y se ha logrado una mejoría significativa del pronóstico en estudios prospectivos ${ }^{30-31}$, pero debe efectuarse en centros especializados y está aún en investigación.

\section{RIESGO MATERNO}

Las complicaciones maternas son significativamente más frecuentes que en embarazos únicos y tienen un mayor incremento en madres tratadas con TRA. Se agrupan en cardiopulmonares (edema agudo de pulmón, pre-eclampsia, por tocólisis); gastrointestinales (hiperemesis grave, hígado graso agudo, colestasis) hematológicas (anemias); obstétricas (operación cesárea, infección puerperal, prematurez, desprendimiento, hemorragias); psicológicas (depresión post-parto), y morbilidad materna por manejo médico (reposo u hospitalización prolongados, trombosis venosa, tocólisis, procedimientos diagnósticos o terapéuticos quirúrgicos invasivos como amniocentesis, 
cerclaje, fetoscopia, coagulación láser, septostomía amniótica).

Hay dos complicaciones maternas de baja incidencia, pero muy graves y con riesgo de muerte materna:

Edema Agudo de Pulmón (EAP): el gemelar es el factor predisponente del $19 \%$ de los casos comunicados de EAP en embarazadas y es una complicación de la tocólisis con betamiméticos $(3 \%)$, de uso frecuente en estas pacientes ${ }^{32}$.

La falla cardíaca se explica por el aumento de volemia propio de gemelar, sobreaporte líquido por tocólisis, efecto de betamiméticos que disminuyen el gasto urinario por estimulación arginina-vasopresina y reninaangiotensina con hipoperfusión renal. Los esteroides comúnmente indicados en la profilaxis del distress repiratorio del prematuro son un factor aditivo.

La prevención del EAP implica identificar en las pacientes que están en riesgo los factores predisponentes: gestación gemelar menor de 30 semanas, edad mayor 30 años, anemia y tocólisis prolongada.

Hígado Graso Agudo (HGA): Valdés comunica una frecuencia local de HGA de $1 / 2834$ partos $^{33}$, mayor que la conocida en la población hispana y plantea una predisposición étnica; la asociación con gemelar se explicaría porque la mayor concentración de estrógenos y ácidos grasos que en únicos, aumentaría el estréss oxidativo intracelular hepático excediendo la capacidad mitocondrial en pacientes susceptibles.

En la revisión de cinco series institucionales del período 1980-200133-37 encontramos ocho gemelares asociados a 76 HGA y la incidencia es de $10.5 \%$.

La asociación tiene en estas pacientes un significado de alto riesgo porque la mortalidad del HGA es aún de 20\%. En el cuidado médico del gemelar deben considerarse su diagnóstico temprano, la interrupción oportuna del embarazo y el manejo interdisciplinario porque son los factores determinantes de la menor mortalidad actual del HGA.

EAP e HGA, ambos de baja incidencia, son más frecuentes en gemelos y es probable que muertes por estas causas se hayan asignado erróneamente a otra posición en el Registro Causas de muerte materna.

En las revisiones históricas de mortalidad materna, el gemelar no aparece considerado como un factor de riesgo, pero actualmente en Europa el riesgo de muerte materna entre gemelos y únicos es de 14.9 versus 5.9 por 100.000 nacimientos y en América Latina es dos es a uno 38,39 .

\section{Múltiples de alto rango (embarazo} multifetal) $\mathrm{Su}$ ocurrencia espontánea es excepcional, pero son muy frecuentes en madres tratadas con TRA y son en la actualidad un problema epidemiológico mayor por el riesgo extremo de prematurez y el alto costo de su manejo.

El mayor incremento es de triples que tienen prematurez y bajo peso excepcionalmente altos: el $41 \%$ nacen antes de semana 33, el 31\% nacen con peso menor a $1500 \mathrm{~g}$ y los prematuros bajo $1000 \mathrm{~g}$ son diez veces más frecuente que en la población general ${ }^{40}$.

En el embarazo multifetal el incremento en el daño neurológico es significativo porque es exponencial según número de fetos ${ }^{41}$,y en los casos de fertilización in vitro aumenta 4 veces y se estima que el múltiple de alto rango contribuye anualmente con el $8 \%$ de los casos de Parálisis Cerebral en EE.UU41-42.

Tabla 3. MPN en Múltiples de Alto Rango.

\begin{tabular}{|ll|}
\hline Triples & $: 109 / 1000(46)$ \\
Cuádruples & $: 116 / 1000(46)$ \\
Quíntuples & $: 253 / 1000(47)$ \\
\hline
\end{tabular}

Aún cuando los embarazos múltiples con TAR son principalmente policigotos, sin embargo se asocian con una división cigótica ${ }^{43}$ y en casuísticas con transferencia de un embrión único la frecuencia de división cigótica es 2.3\%, seis veces mayor que la espontánea $(0.4 \%)$. Su 
importancia clínica es la alta morbilidad y mortalidad asociadas a la gestación monocigota.

Otros riesgos observados del multifetal son malformaciones, defectos cromosómicos y musculoesqueléticos después de la inyección intracitoplasmática de espermios (ICSI) y con la fertilización in vitro ${ }^{44}$, aún cuando en este hallazgo pueden influir factores parentales ${ }^{45}$.

La Mortalidad Perinatal, alta y progresiva de la pluralidad, se observa en Tabla 3.

Las madres presentan complicaciones graves hasta en el 40\% de los casos: pre-eclamsia, eclampsia, HELLP, hígado graso agudo, hipertensión, porque impone una adaptación materna que sobrepasa su capacidad fisiológica, aumentando el riesgo de muerte $5,38,39$.

Pese a la satisfacción de las parejas infértiles hay controversia por las complicaciones maternas y perinatales de los embarazos multifetales, por el problema ético de la manipulación de embriones (criopreservación de cigoto y conceptus), por la discriminación en la selección embrionaria y porque aquellos no utilizados no tienen opción de sobrevivir.

El significado de estas complicaciones iatrogénicas ha reorientado actualmente la terapia de la infertilidad a la prevención de la concepción múltiple, mediante la transferencia de un solo embrión ${ }^{48}$ o la obtención de uno o dos foliculos en ovulación inducida, restringiendo el concepto de éxito a la obtención de un embarazo único. En informe reciente del Registro Chileno de Reproducción Asistida ${ }^{49}$ se demuestra una disminución de la media de embriones transferidos de 3.3 en 1999 a 2.7 en 2002, con una disminución de los embarazos de Alto Rango de 13.4 a $6.2 \%$, lo que expresa un esfuerzo por reducir esta complicación, pero la tasa global de embarazo múltiple, que incluye gemelos, se ha mantenido estable.

Como política de Salud, para los casos en que no se logre el objetivo del embarazo único es necesaria la cobertura asistencia y económica, por el alto riesgo descrito.

Además de la prevención, en la red asistencial está implícita la atención de alta complejidad obstétrica y neonatal, con procedimientos de

\section{REFERENCIAS}

1.- KIELY JL, KIELY M. Epidemiologic trends in mùltiple birth in the United States.1971-1998. Twins Res 2001, 4: 132-133.

2.- LOOS R, DEROM C, VLIETINCK R, DEROM R. The East Flanders Prospective Twin Survey (Belgium): a population-based register. Twin Res 1998, 1: 167-195.

3.-NYGREN KG, ANDERSEN AN. Assisted reproductive technology in Europe, 1999. Results generated from European Registers by ESHRE. Human Reprod 2002, 17: 3260-3274.

4.- BLICKSTEIN I, GOLDMAN RD, MAZKERETH $R$. Incidence and birth weight characteristics of twin born to mothers aged 40 years or more compared with 35-39 years old mothers: a population study. J Perinat Med 2001, 29: 126-132.

5.-SAUER MV, PAULSON RJ, LOBO RA. Oocyte donation to women of advanced reproductive age : pregnancy reesults and obstetrical outcomes in patients 45 years and older. Human Reprod. 1996, 11 : 2540-2543.

6.- GARDNER MO, GOLDENBERG R, CLIVER S, TUCKER J, NELSON K, COOPER R. The origin and the outcome of preterm twin or singleton. Obstet Gynecol 1994, 85: 553.

7.- KIELY JL. The epidemiology of perinatal mortality in múltiple births. Bull N Y Acad Med 1990, 66: 618-37.

8.- SPELLACY WN, HANDLER H, FERRE CD. A case control study of 1253 twin pregnancies from a 19821987 perinatal data base. Obstet Gynecol 1990, 75: 168-171.

9.- OHEL G, GRANAT M, ZEEVI D, GOLAN A, WEXLER S, DAVID MP, SCHENKER JG. Advanced ultrasonic placental maturation in twins pregnancies. AJOG 1987, 156:76-78

10.- BLECKER OP, BREUR W, HUIDEKOPER BL. A study of birthweight, placental weight and mortality of twins as compared to singleton. Br J Obstet Gyneacol 1979, 86:111-118.

11.- LUKE B, MINOGUE J, WITTER FR, KEITH LG, JONSON TR. The ideal twin pregnancy: patterns of weight gain, discordancy, and length of gestation AJOG 1993 169:588-597.

12.- POWER WF, KIELY JL. The risks confronting twins: a national perspective. Am J Obstet Gynecol 1994, 170:456-461.

13.- GOLDENBERG RL, IAMS J, MIODOVNIK M, VAN DORSTEN JP, THURNAU G, BOTTOM S, et al. The preterm prediction study: risk factors in twins gestations. Am J Obstet Gynecol 1996, 175: 1047-1053.

14.- GHAI V, VIDYASAGAR D. Morbility and mortality factors in twin: an epidemiologic approach. Clin Perinatol 1988, 15:123-140. 
15.-ROUSE DJ, SKOPEC GS, ZLANTNIK FJ. Fundal height as predictor of preterm twin delivery. Obstet Gynecol 1993, 81:211.

16.-ROMERO R, SHAMMA F, AVILA C, JIMÉNEZ C, CALLAHAN R, NORES J. Infection and labor VI. Prevalence, microbiology and clinical significance of intraamniotic infection in twin gestation with preterm labor. Am J Obstet Gynecol 1990, 163:757.

17.-MENARD RK, NEWMAN R, KEENAN R. Prognostic significance of prior preterm delivery: Twins or Singleton. AJOG 1995, 172:257.

18.-LA PLAZA J, ROOT L, TASSANAWIPASA, CERVERA P. Cerebral palsy in twins. Dev Med Child Neurol 1992, 34:1053-63.

19.-PETTERSON B, NELSON KB, WATSON L, STANLEY F. Twins, Triplets and cerebral palsy in birth in Western Australia in the 1980s. BMJ 1993, 307: 1239-43.

20.- YOKOYAMA Y, SHIMIZU T, HAYAKAWA K. Prevalence of cerebral palsy in twins, triplets and quadruplets. Int J Epidemiol 1995, 24: 943-48.

21.- WILLIAMS K, HENNNESSY E, ALBERNAN B. Cerebral pals y: effects of twining, birthweight and gestational age. Arch Dis Child 1996, 75: F178-82.

22.-GRONBERG H, DAMBER L, DAMBER JE. Studies of genetic factors in prostate cancer in a twin population. J Urol 1994, 152 ( 5 Ptl): 1484-1487.

23.-SUMETHKUL V, JIRASIRITHAM S, SURA T. Renal transplantation between identical twins: The application of reciprocal full-thickness skin grafts as a guideline for antirejection therapyTranspl Proc. 1994, 26: 2141-2142.

24.-SEBIRE NJ, SNIJDERS RJM, HUGHES K, SEPULVEDA W, NIKOLAIDES KH. The hidden mortality of monochorionic twin pregnancies. $\mathrm{Br} \mathrm{J}$ Obstet Gynecol 1997, 104: 1203-1207.

25.-BERNIRSCHKE K. The contribution of placental anastomoses to prenatal twin damage. Hum Pathol 1992, 23: 1319-1320.

26.-BAJORIA R, WIGGLESWORTH J, FISK N M. Angioarchitecture of monochorionic placentas in relation to the twin-twin tranfusion syndrome. Am J Obstet Gynecol 1995, 172: 855- 863.

27.-MINAKAMI H, HONMA Y, MATSUBARA S, USHIDA A, SHIRAISHI H, SATO I. Effects of placental chorionicity on outcome in twin pregnancy. J Reprod Med 1999, 44: 595-600.

28.-MAIER RF, BIALOBREZESKY B, GROSSA, VOGEL M, DUDDEENHAUSEN JW, OBLADEN $\mathrm{M}$. Acute and cronic fetal hypoxia in monochorionic and dichorionic twins. Obstet Gynecol 1995, 86: 973977.

29.-RODRÍGUEZ JG, PORTER H, SIRRTA GM, SOOTHILL PW. Twin to twin tranfusion in a dichorionic pregnancy without the oligohydramniospolyhydramnios sequense. Br J Obstet Gynecol 1996, 103:1056.
30.- GRATACÓS E, VAN SCHOUBROECK D, CARRERAS E, DEVLIEGER R, ROMA E, CABERO $L$ et al. Impact of laser coagulation in severe twin-twin tranfusion syndrome on fetal Doppler indices and venous blood flow volume. Ultrasound Obstet Gynecol 2002, 20: 125-130.

31.- DE LIA JE, KUHLMAN RS, LÓPEZ KP. Treating previable twin-twin tranfusion syndrome with fetoscopic laser surgery: outcomes following the learning curve. J Perinat Med 1999, 27: 61-67.

32.- HUDGENS DR, CONRADI SE. Sudden death associated with terbutaline sulfate administration. Am J Obstet Gynecol 1993, 169: 120-121.

33.- VALDÉS E, OKSENBERG D, CORTÉS C. Hígado graso agudo del embarazo: estudio retrospectivo clínico-patológico de 10 casos. Rev Chil de Obstetricia y Ginecología 2001, 66, 4: 295-305.

34.- DAVIES M, WILKINSON SP, HANIS MA, PORTMAN B, BRUDENELL JM, NEWTON JR et al. Acute liver disease with encephalopathy and renal failure in late pregnancy and early puerperium: a study of fourteen patients. Br J Obstet Gynaecol 1980, 87: 1005.

35.- CASTRO M, FASSETT M, REYNOLDS T, SHAW K, GOODWIN T. Reversible peripartum liver failure: A new perspective on the diagnosis, treatment and cause of acute fatty liver of pregnancy, based on 28 consecutive cases. Am J Obstet Gynecol 1999, 181(2): $389-395$.

36.- POCKROS PJ, PETERS RL, REYNOLDS TB. Idiophatic fatty liver of pregnancy: findings in ten cases. Medicine. 1984, 63: 1-11.

37.- REYES H. SANDOVAL L, WAINSTEIN A. RIBALTA J, DONOSO S, SMOCK G et al. Acute fatty liver of pregnancy: a clinical study of 12 episodes in 11 patients. Gut 1994, 35: 101-106.

38.- SENAT MV, ANCEL PY, BOUVIER-COLLE MH, BREART G. How does multiple pregnancy affect maternal mortality and morbidity?. Clin Obstet Gynecol 1998, 41: 79-83.

39.- CONDE-AGUDELO A, BELIZAN J. Maternal mortality and associated with multiple pregnancy. Twins Res 1999, 2:S3.

40.- BLICKSTEIN I, JAQUES DL. The Maria triplet database:1988-2000. In Keith LG, Blickstein I, editors. Triplet pregnancies and their consequences. New York: The Partenon Publishing Group. 2002, 267-291.

41.- KIELY JL, KIELY M, BLICKSTEIN I. Contribution of the rise in multiple birth to a potential increase in cerebral palsy. Peditr Res 2000, 47: 314 A.

42.- STROMBERG B, DAHLQUIST G, ERICSON A, FINNSTROM O, KOSTER M, STJERNQVIST K. Neurological sequelae in children born after in vitro fertilization: a population-based study. Lancet 2002, 359: 461-465.

43.- BLICKSTEIN I, VERHOEVEN HC, KEITH LG. Zigotic splitting after assisted reproduction. $N$ England J Med 1999, 340: 738-739. 
44.-HANSEN M, KURINCZUK JJ, BOWER C, WEBB $\mathrm{S}$. The risk of major birth defects after intracytoplasmatic sperm injection and in vitro fertilization. N England J Med 2002, 346: 725-730.

45.-LUDWIG M, KATALINIC A. Malformation rate in fetus and children conceived after ICSI: results of a prospective cohort study. Reprod Biomed Online 2002, 5: 171-178.

46.-NEWMAN RB. Management of triplets and other high order multiples. In: Newman RB,

Luke B, editors. Multifetal pregnancy. Philadelphia:

Lippincott Williams and Wilckins; 2000: 192-219.
47.- FRANCOIS K, ALPERIN A, ELLIOT JP. Outcome of quintuplet pregnancies. J Reprod Med 2001, 46: 1047-1051.

48.- HAMBERGER L, HAZEKAMPJ. Towards single embryo transfer in IVF. J. Reprod Immunol 2002, 55: 141-148.

49.- BALMACEDA J, GALDAMES V. Registro Chileno de Reproducción Asistida 2002. Editorial.

Usted puede comentar éste y otros artículos publicados en la Revista Chilena de Salud Pública, enviando un correo electrónico a revistasp@med.uchile.cl 